\title{
Diffusion phenomena for partially dissipative hyperbolic systems
}

\author{
Jens Wirth ${ }^{\mathrm{a}, 1}$ \\ ${ }^{a}$ Mathematisches Institut, LMU München, Theresienstr. 39/1, 80339 München \\ Institut für Analysis, Dynamik und Modellierung, Universität Stuttgart, Pfaffenwaldring 57, 70569 Stuttgart
}

\begin{abstract}
We consider a partially dissipative hyperbolic system with time-dependent coefficients and show that under natural assumptions its solutions behave like solutions to a parabolic problem modulo terms of faster decay.

This generalises the well-known diffusion phenomenon for damped waves and gives some further insights into the structure of dissipative hyperbolic systems.
\end{abstract}

Keywords: hyperbolic systems, partial dissipation, uniform Kalman rank condition, diffusion phenomenon

2010 MSC: 35L05, 35L15

\section{Introduction}

The classical diffusion phenomenon observed by Hsiao-Liu [3] and Nishihara [1] provides an asymptotic equivalence for solutions of the damped wave equation and corresponding solutions of the heat equation. We will recall the main results first before explaining our generalisations. If one considers the Cauchy problem for the damped wave equation

$$
u_{t t}-\Delta u+u_{t}=0, \quad u(0, \cdot)=u_{0}, \quad u_{t}(0, \cdot)=u_{1}
$$

on $\mathbb{R}^{n}$ together with the associated heat equation

$$
w_{t}=\Delta w, \quad w(0, \cdot)=w_{0}=u_{0}+u_{1}
$$

it is known that their solutions satisfy

$$
\|u(t, \cdot)-w(t, \cdot)\|_{2} \leq C t^{-1}\left(\left\|u_{0}\right\|_{2}+\left\|u_{1}\right\|_{2}\right)
$$

and corresponding estimates for higher order derivatives or in different $L^{p}$ norms. Note, that both solutions $u$ and $w$ do not decay in general. In this sense, the above estimate justifies to treat the solution of the heat equation as main term of the solution of the damped wave equation.

The above estimate is not the original one. There has been progress over the recent years to improve rates and constants, we refer only to a brief selection of papers. Nishihara [12] and

\footnotetext{
Email address: jens.wirth@mathematik.uni-stuttgart.de (Jens Wirth)

${ }^{1}$ The research presented in this paper was partially supported by the German Science Foundation (DFG) with grant WI 2064/5-1.
} 
Narazaki [10] extended the results to $L^{p}-L^{q}$ estimates, Han-Milani [9] showed (independently) $L^{1}$ estimates for solutions. There have been extensions by Ikehata-Nishihara [6] and Chill-Hareaux [2] to abstract evolution equations. Recently Radu-Todorova-Yordanov [13] used a different line of thought to estimate the difference of solutions in terms of the heat semigroup acting on the initial data. Their method allows to show

$$
\|u(t, \cdot)-w(t, \cdot)\|_{2} \leq C t^{-1}\left(\left\|\mathrm{e}^{t \Delta / 2} u_{0}\right\|_{2}+\left\|\mathrm{e}^{t \Delta / 2} u_{1}\right\|_{2}\right)+\mathrm{e}^{-t / 16}\left(\left\|u_{0}\right\|_{2}+\left\|u_{1}\right\|_{H^{-1}}\right)
$$

for $t \geq 1$ and all initial data such that the norms on the right hand side are finite. Estimates of this form are valuable to the study of nonlinear damped wave equations and allow to show that critical exponents and associated global in time solutions in the supercritical cases are governed by the parabolic problem.

The author of this note extended the diffusion phenomenon to time-dependent dissipation terms in [16], see also [18] for an overview of the results. There one considers the wave equation

$$
u_{t t}-\Delta u+b(t) u_{t}=0, \quad u(0, \cdot)=u_{0}, \quad u_{t}(0, \cdot)=u_{1}
$$

with (for simplicity monotone) smooth and positive coefficient function $b=b(t)$ subject to estimates

$$
\left|\partial_{t}^{k} b(t)\right| \leq C_{k} b(t)\left(\frac{1}{1+t}\right)^{k}
$$

Such a dissipation term is called effective if $\lim _{t \rightarrow \infty} t b(t)=\infty$ and allows for a diffusion phenomen if in addition $1 / b^{3} \notin \mathrm{L}^{1}\left(\mathbb{R}_{+}\right)$. Under this assumption solutions to the Cauchy problem (1.5) behave asymptotically like solutions to the heat equation

$$
w_{t}=\frac{1}{b(t)} \Delta w, \quad w(0, \cdot)=w_{0}=u_{0}+\mu u_{1}, \quad \mu=\int_{0}^{\infty} \exp \left(-\int_{0}^{t} b(s) \mathrm{d} s\right) \mathrm{d} t .
$$

This paper is not concerned with scalar second order equations. In the sequel we will consider hyperbolic systems with time-dependent coefficients. The situation without dissipation was treated by the author in [14], [15] and allowed to derive dispersive estimates for solutions. On the other hand, a fully dissipative hyperbolic system will have exponential decay of its energy and is of less interest for us. We are going to study hyperbolic systems with a partial dissipation, where only one of the modes of the solution is damped directly while all the others decay due to coupling effects. Before formulating the problem we need to introduce some notation. We denote by $\mathcal{T}\{\ell\}$ the set of all smooth complex-valued functions $f \in \mathrm{C}^{\infty}([0, \infty) ; \mathbb{C})$ subject to estimates

$$
\left|\partial_{t}^{k} f(t)\right| \leq C_{k}\left(\frac{1}{1+t}\right)^{\ell+k}
$$

We further denote by $\mathcal{T}\{-\infty\}=\bigcap_{\ell} \mathcal{T}\{\ell\}$ the set of rapidly decaying functions, it coincides with the Schwartz space $\mathcal{S}([0, \infty))$ on the half-line. Then we consider hyperbolic systems of the form

$$
\mathrm{D}_{t} U=\sum_{k=1}^{n} A_{k}(t) \mathrm{D}_{x_{k}} U+\mathrm{i} B(t) U, \quad U(t, \cdot)=U_{0} \in \mathcal{S}\left(\mathbb{R}^{n} ; \mathbb{C}^{d}\right),
$$


$\mathrm{D}_{t}=-\partial_{t}$ being the Fourier derivative. Main difference to the considerations in [14], [15] is that we do not assume $B(t)$ to be of lower order in the $\mathcal{T}$-hierarchy. We assume that

$$
A_{k}(t), B(t) \in \mathcal{T}\{0\} \otimes \mathbb{C}^{d \times d}
$$

Furthermore, we will make three main assumptions.

(B1) The matrices $A_{k}(t)$ are self-adjoint and $\Re B(t)=\frac{1}{2}\left(B(t)+B(t)^{*}\right) \geq 0$ is non-negative.

(B2) The matrices $B(t)$ are singular in the sense that $\operatorname{det} B(t)=0$ and

$$
\operatorname{dist}(0, \Re \operatorname{spec} B(t) \backslash\{0\}) \geq \kappa>0
$$

uniformly in $t \geq t_{0}$ for some positive constant $t_{0}$. The eigenvalue 0 is assumed to be simple.

(B3) The matrices $A(t, \xi)=\sum_{k=1}^{n} A_{k}(t) \xi_{k}$ and $B(t)$ satisfy for all $\nu \in \mathbb{C}^{d}$

$$
\frac{1}{c}\|\nu\|^{2} \leq \sum_{j=0}^{d-1} \epsilon_{j}\left\|B(t)(A(t, \xi))^{j} \nu\right\|^{2} \leq c\|\nu\|^{2}, \quad t \geq t_{0},
$$

for any choice of numbers $\epsilon_{0}, \ldots, \epsilon_{d-1}>0$ and suitable constants $c$ and $t_{0}$ depending on them.

Assumption (B1) guarantees that the system is symmetric hyperbolic and (partially) dissipative. Therefore, the energy estimate

$$
\|U(t, \cdot)\|_{L^{2}} \leq\left\|U_{0}\right\|_{L^{2}}
$$

is valid and implies in particular existence and uniqueness of solutions. By assumption (B2) we know that one mode is not dissipated, while assumption (B3) will be used to show that the high frequency parts of solutions are still exponentially decaying. Inspired by Beauchard-Zuazua [1], we will refer to (B3) as uniform Kalman rank condition. If $A_{k}$ and $B$ are independent of $t$ it just means that

$$
\operatorname{rank}\left(B|A(\xi) B| \cdots \mid A(\xi)^{d-1} B\right)=d,
$$

which is the classical Kalman rank condition arising in the control theory of ordinary differential systems. Under certain natural assumptions, this is equivalent to the algebraic condition of Kawashima-Shizuta [8], but the latter are more complicated to rewrite uniformly depending on parameters.

This paper is organised as follows. In section 2 we will develop a block-diagonalisation scheme for small frequencies in order to conclude asymptotic information about solutions. It is based on assumptions (B1) and (B2). Later on in section 4 we will sketch the decay estimates for large frequencies based on assumption (B3) and Lyapunov functionals. Finally, section 5 gives decay estimates of solutions combined with comparison statements to corresponding parabolic problems.

\section{Diagonalisation for small frequencies}

Symbol classes. The diagonalisation procedure is based on ideas from [7] and [17] and applies to the elliptic zone

$$
\mathcal{Z}_{\text {ell }}(c)=\left\{(t, \xi):|\xi| \leq c, t \geq c^{-1}\right\}
$$


of the extended phase space $[0, \infty) \times \mathbb{R}^{n}$. It consists of small frequencies for large times. All considerations will be done for $t \geq t_{0}$ with $t_{0}$ sufficiently large. This ensures uniform block-diagonalisability of the matrix $B(t)$, i.e., due to assumption (B3) we find a matrix-valued function

$$
M(t) \in \mathcal{T}\{0\} \otimes \mathbb{C}^{d \times d}
$$

with uniformly bounded inverse such that

$$
M^{-1}(t) B(t) M(t)=\mathcal{D}(t)=\left(\begin{array}{cc}
0 & \\
& \tilde{\mathcal{D}}(t)
\end{array}\right)
$$

and $\tilde{\mathcal{D}}(t)$ has eigenvalues with imaginary parts in $[\kappa, \infty)$. As $B(t)$ is the main term for small frequencies, this allows to asymptotically decouple the system in a sufficiently small zone $\mathcal{Z}_{\text {ell }}(c)$. For this we introduce the symbol classes

$$
\mathcal{P}\{m\}=\left\{p(t, \xi)=\sum_{|\alpha| \leq m} p_{\alpha}(t) \xi^{\alpha}: p_{\alpha}(t) \in \mathcal{T}\{m-|\alpha|\}\right\}
$$

consisting of polynomials with coefficients in the $\mathcal{T}$-classes. Symbols from these classes can be thought of as homogeneous components, we use

$$
\mathcal{P}_{\geq}\{m\}=\left\{\sum_{k=m}^{\infty} p_{k}(t, \xi): p_{k}(t, \xi) \in \mathcal{P}\{k\}\right\}
$$

to denote symbols which are (uniformly) convergent power series in $\mathcal{Z}_{\text {ell }}(c)$ for $c$ sufficiently small. A first transformation. Denoting $V^{(0)}(t, \xi)=M^{-1}(t) \widehat{U}(t, \xi)$, we obtain the equivalent system

$$
\mathrm{D}_{t} V^{(0)}=\left(\mathcal{D}(t)+\sum_{k=1}^{n} M^{-1}(t) A_{k}(t) M(t) \xi_{k}+\left(\mathrm{D}_{t} M^{-1}(t)\right) M(t)\right) V^{(0)}
$$

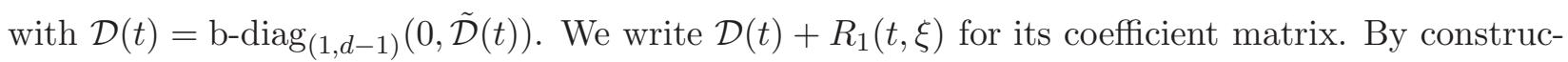
tion, the matrix $R_{1}(t, \xi) \in \mathcal{P}\{1\} \otimes \mathbb{C}^{d \times d}$ is asymptotically of lower order.

The diagonalisation hierarchy. We start with the system

$$
\mathrm{D}_{t} V^{(0)}=\left(\mathcal{D}(t)+R_{1}(t, \xi)\right) V^{(0)}
$$

with $R_{1}(t, \xi) \in \mathcal{P}\{1\}$. Before setting up the complete hierarchy, we will discuss its first step. We construct a matrix $N^{(1)}(t, \xi) \in \mathcal{P}\{1\} \otimes \mathbb{C}^{d \times d}$ such that

$$
\left(\mathrm{D}_{t}-\mathcal{D}(t)-R_{1}(t, \xi)\right)\left(\mathrm{I}+N^{(1)}(t, \xi)\right)-\left(\mathrm{I}+N^{(1)}(t, \xi)\right)\left(\mathrm{D}_{t}-\mathcal{D}(t, \xi)-F_{1}(t, \xi)\right) \in \mathcal{P}\{2\} \otimes \mathbb{C}^{d \times d}
$$

holds true for some block-diagonal matrix $F_{1}(t, \xi) \in \mathcal{P}\{1\} \otimes \mathbb{C}^{d \times d}$. Collecting all terms not belonging to the right class yields again conditions for the matrices $N^{(1)}(t, \xi)$ and $F_{1}(t, \xi)$. Indeed,

$$
\left[\mathcal{D}(t), N^{(1)}(t, \xi)\right]=-R_{1}(t, \xi)+F_{1}(t, \xi)
$$


must be satisfied and, therefore, we set

$$
F_{1}(t, \xi)=\mathrm{b}-\operatorname{diag}_{(1, d-1)} R_{1}(t, \xi),
$$

b-diag denoting the block-diagonal part to the partition of indices2, combined with

$$
N^{(1)}(t, \xi)=\left(\begin{array}{cc}
0 & \tilde{N}_{1,1}(t, \xi)^{\top} \\
\tilde{N}_{1,2}(t, \xi) & 0
\end{array}\right), \quad \tilde{N}_{1, j}(t, \xi)=\int_{0}^{\infty} \mathrm{e}^{-s \tilde{\mathcal{D}}(t)} \tilde{R}_{1, j}(t, \xi) \mathrm{d} s
$$

for

$$
R_{1}(t, \xi)-F_{1}(t, \xi)=\left(\begin{array}{cc}
0 & \tilde{R}_{1,1}(t, \xi)^{\top} \\
\tilde{R}_{1,2}(t, \xi) & 0
\end{array}\right)
$$

Due to the spectral properties of $\tilde{\mathcal{D}}(t)$ the above integral converges and it is a matter of direct calculation to check that $N^{(1)}$ indeed satisfies the Sylvester equation (2.9). For further details see 17]. As desired, the construction implies $N^{(1)}(t, \xi), F_{1}(t, \xi) \in \mathcal{P}\{1\} \otimes \mathbb{C}^{d \times d}$. The construction generalises to remainders from better classes.

Lemma 2.1. Assume (B1) and (B2) are satisfied and let $k \in \mathbb{N}, k \geq 1$. Then there exists a constant $c_{k}$ and matrices $N_{k}(t, \xi) \in \mathcal{P}_{\geq}\{0\} \otimes \mathbb{C}^{d \times d}$, block-diagonal matrices $F_{k}(t, \xi) \in \mathcal{P}_{\geq}\{1\} \otimes \mathbb{C}^{d \times d}$ and matrices $R_{k+1}(t, \xi) \in \mathcal{P}_{\geq}\{k+1\} \otimes \mathbb{C}^{d \times d}$ such that

$$
\left(\mathrm{D}_{t}-\mathcal{D}(t)-R_{1}(t, \xi)\right) N_{k}(t, \xi)=N_{k}(t, \xi)\left(\mathrm{D}_{t}-\mathcal{D}(t)-F_{k}(t, \xi)-R_{k+1}(t, \xi)\right)
$$

holds true within $\mathcal{Z}_{\mathrm{ell}}\left(c_{k}\right)$. Furthermore, $N_{k}(t, \xi)$ is uniformly invertible within this zone.

Proof. We construct recursively the matrices $N^{(k)}(t, \xi) \in \mathcal{P}\{k\} \otimes \mathbb{C}^{d \times d}$ and $F^{(k)}(t, \xi) \in \mathcal{P}\{k\} \otimes \mathbb{C}^{d \times d}$ block-diagonal, such that for

$$
N_{K}(t, \xi)=\mathrm{I}+\sum_{k=1}^{K} N^{(k)}(t, \xi), \quad F_{K}(t, \xi)=\sum_{k=1}^{K} F^{(k)}(t, \xi),
$$

the estimate

$$
B_{K}(t, \xi)=\left(\mathrm{D}_{t}-\mathcal{D}(t)-R_{1}(t, \xi)\right) N_{K}(t, \xi)-N_{K}(t, \xi)\left(\mathrm{D}_{t}-\mathcal{D}(t)-F_{K}(t, \xi)\right) \in \mathcal{P}_{\geq}\{K+1\}
$$

is valid. We just did this for $K=1$, it remains to do the recursion $k \mapsto k+1$. Assume $B_{k}(t, \xi) \in$ $\mathcal{P}\{k+1\} \otimes \mathbb{C}^{d \times d}$. The requirement to be met is that

$$
B_{k+1}(t, \xi)-B_{k}(t, \xi)=-\left[\mathcal{D}(t), N^{(k+1)}(t, \xi)\right]+F^{(k+1)}(t, \xi) \quad \bmod \mathcal{P}_{\geq}\{k+2\} \otimes \mathbb{C}^{d \times d}
$$

for block-diagonal $F^{(k+1)}(t, \xi)$, which yields

$$
F^{(k+1)}(t, \xi)=-\mathrm{b}-\operatorname{diag}_{(1, d-1)} B_{k}(t, \xi)
$$

\footnotetext{
${ }^{2}$ For a partition $\Pi$ of $\{1, \ldots, d\}$ and a matrix $A$ we denote by b- $\operatorname{diag}_{\Pi} A$ the block-diagonal matrix with the same entries on the block diagonal, see [17]. If partitions consist of sets of adjacent numbers, we denote them as tuple $\Pi=\left(d_{1}, \ldots, d_{m}\right), d_{1}+\cdots+d_{m}=d$, encoding their sizes.
} 
together with

$$
N^{(k+1)}(t, \xi)=\left(\begin{array}{cc}
0 & \tilde{N}_{k+1,1}(t, \xi)^{\top} \\
\tilde{N}_{k+1,2}(t, \xi) & 0
\end{array}\right), \quad \tilde{N}_{k+1, j}(t, \xi)=-\int_{0}^{\infty} \mathrm{e}^{-s \tilde{\mathcal{D}}(t)} \tilde{B}_{k, j}(t, \xi) \mathrm{d} s
$$

for the components

$$
B_{k}(t, \xi)+F_{1}(t, \xi)=\left(\begin{array}{cc}
0 & \tilde{B}_{k, 1}(t, \xi)^{\top} \\
\tilde{B}_{k, 2}(t, \xi) & 0
\end{array}\right) .
$$

It is evident that the construction implies $F^{(k+1)}(t, \xi), N^{(k+1)}(t, \xi) \in \mathcal{P}\{k+1\} \otimes \mathbb{C}^{d \times d}$ together with $B_{k+1}(t, \xi) \in \mathcal{P}_{\geq}\{k+2\} \otimes \mathbb{C}^{d \times d}$.

The matrices $N_{k}(t, \xi) \in \mathcal{P}_{\geq}\{0\} \otimes \mathbb{C}^{d \times d}$ are invertible with inverse $N_{k}^{-1}(t, \xi) \in \mathcal{P}_{\geq}\{0\} \otimes \mathbb{C}^{d \times d}$ if we restrict our consideration to a sufficiently small elliptic zone $\mathcal{Z}_{\text {ell }}\left(c_{k}\right)$. The latter follows by using the Neumann series for the inverse. The result of the above consideration can be summarised in the following lemma.

It is worth having a closer look at the upper-left corner entry of the matrix $F_{k}(t, \xi)$ and consequences for them based on assumption (B2). The above lemma implies that modulo $\mathcal{P}_{\geq}\{3\}$ the entry is of the form

$$
f_{1}^{(k)}(t, \xi)=\mathrm{i} \sum_{i, j=1}^{d} \alpha_{i, j}(t) \xi_{i} \xi_{j}+\sum_{i=1}^{d} \beta_{i}(t) \xi_{i}+\gamma(t) \quad \bmod \mathcal{P}_{\geq}\{3\}
$$

with $\alpha_{i, j}(t) \in \mathcal{T}\{0\}, \beta_{i}(t) \in \mathcal{T}\{0\}$ and $\gamma(t) \in \mathcal{T}\{1\}$. On the other hand, modulo $\mathcal{O}\left(t^{-1}\right)$ the eigenvalues of $F_{k}(t, \xi)$ and of $A(t, \xi)$ coincide. As (B2) is a spectral condition implying that 0 is a local (quadratic) minimum of an eigenvalue branch contained in the complex upper half-plane, some terms in (2.20) have to vanish. In particular we see that $\beta_{i}(t)$ has to decay, $\beta_{i}(t) \in \mathcal{T}\{1\}$, and also that the real part of the quadratic matrix $\left(\alpha_{i, j}(t)\right)_{i, j}$ is positive definite modulo $\mathcal{T}\{1\}$. The latter is a direct consequence of the non-degeneracy of that minimum.

Corollary 2.2. Assume (B1) and (B2) and let $k \geq 1$. Then modulo $\mathcal{P}_{\geq}\{3\}$ the upper-left corner entry of $F_{k}(t, \xi)$ satisfies

$$
f_{1}^{(k)}(t, \xi)=\mathrm{i} \xi^{\top} \boldsymbol{\alpha}(t) \xi+\boldsymbol{\beta}(t)^{\top} \xi+\gamma(t) \quad \bmod \mathcal{P}_{\geq}\{3\}
$$

with $\boldsymbol{\alpha}(t) \in \mathcal{T}\{0\} \otimes \mathbb{C}^{d \times d}$ having positive definite real part uniform in $t \geq t_{0}$ for $t_{0}$ sufficiently large, $\boldsymbol{\beta}(t) \in \mathcal{T}\{1\} \otimes \mathbb{C}^{d}$ and $\gamma(t) \in \mathcal{T}\{1\}$.

If the matrix $B(t)$ is self-adjoint for all $t$ then we can choose $M(t)$ unitary and, therefore, the construction gives that $\gamma(t)$ is real-valued modulo $\mathcal{T}\{2\}$. By assumption (B1) in the form of estimate (1.12) we know that $\exp \left(-\Im \int_{t_{0}}^{t} \gamma(\theta) \mathrm{d} \theta\right) \lesssim 1$.

\section{Asymptotic integration and small frequency expansions}

Fundamental solutions. We consider the transformed problem in $V^{(k)}(t, \xi)=N_{k}(t, \xi) V^{(0)}(t, \xi)$,

$$
\mathrm{D}_{t} V^{(k)}(t, \xi)=\left(\mathcal{D}(t)+F_{k}(t, \xi)+R_{k+1}(t, \xi)\right) V^{(k)}(t, \xi)
$$


and reformulate this as integral equation for its fundamental solution $\mathcal{E}_{k}(t, s, \xi)$, i.e., the matrixvalued solution to initial data $\mathcal{E}_{k}(s, s, \xi)=\mathrm{I} \in \mathbb{C}^{d \times d}$. Let for this $\Theta_{k}(t, s, \xi)$ be the fundamental solution to the block-diagonal system $\mathrm{D}_{t}-\mathcal{D}(t)-F_{k}(t, \xi)$. Then

$$
\Theta_{k}(t, s, \xi)=\left(\begin{array}{cc}
\Xi_{k}(t, s, \xi) & 0 \\
0 & \tilde{\Theta}_{k}(t, s, \xi)
\end{array}\right), \quad\left\|\tilde{\Theta}_{k}(t, s, \xi)\right\| \lesssim \mathrm{e}^{-\tilde{c}(t-s)}
$$

for $t \geq s$ uniformly within $\mathcal{Z}_{\text {ell }}(\epsilon)$ for $\epsilon \leq c_{k}$ sufficiently small. Here,

$$
\Xi_{k}(t, s, \xi)=\exp \left(\mathrm{i} \int_{s}^{t} f_{1}^{(k)}(\theta, \xi) \mathrm{d} \theta\right)
$$

is uniformly bounded and gives (for $k=2$ ) the fundamental solution to a parabolic problem and $\tilde{\Theta}_{k}(t, s, \xi)$ is exponentially decaying as the fundamental solution of a dissipative system. Furthermore, the matrix $\mathcal{E}_{k}(t, s, \xi)$ satisfies the Volterra integral equation

$$
\mathcal{E}_{k}(t, s, \xi)=\Theta_{k}(t, s, \xi)+\int_{s}^{t} \Theta_{k}(t, \theta, \xi) R_{k+1}(\theta, \xi) \mathcal{E}_{k}(\theta, s, \xi) \mathrm{d} \theta
$$

We solve this equation using the Neumann series

$$
\begin{array}{r}
\mathcal{E}_{k}(t, s, \xi)=\Theta_{k}(t, s, \xi)+\sum_{\ell=1}^{\infty} \mathrm{i}^{\ell} \int_{s}^{t} \Theta_{k}\left(t, t_{1}, \xi\right) R_{k+1}\left(t_{1}, \xi\right) \int_{s}^{t_{1}} \cdots \\
\cdots \int_{s}^{t_{\ell-1}} \Theta_{k}\left(t_{\ell-1}, t_{\ell}, \xi\right) R_{k+1}\left(t_{\ell}, \xi\right) \mathrm{d} t_{\ell} \cdots \mathrm{d} t_{1}
\end{array}
$$

This series converges and and its value can be estimated by

$$
\left\|\mathcal{E}_{k}(t, s, \xi)\right\| \leq \exp \left(\int_{s}^{t}\left\|R_{k+1}(\theta, \xi)\right\| \mathrm{d} \theta\right) .
$$

Based on the estimates for the remainder term $R_{k+1}(t, \xi) \in \mathcal{P}_{\geq}\{k+1\} \otimes \mathbb{C}^{d \times d}$, we even obtain uniform convergence within the smaller zone $\mathcal{Z}_{\text {ell }}\left(c_{k}\right) \cap\left\{t|\xi|^{(k+\overline{1}) / 2} \leq \delta\right\}$ for any constant $\delta$ and $\left\|\mathcal{E}_{k}(t, s, \xi)-\Theta_{k}(t, s, \xi)\right\| \rightarrow 0$ as $c_{k} \rightarrow 0$ for fixed $\delta>0$ as soon as we choose $k \geq 1$.

In particular we obtain a pointwise bound in terms of $\Xi_{k}(t, s, \xi)$ for $t|\xi|^{2} \leq \delta$.

Lemma 3.1. Assume (B1), (B2) and let $k \geq 2$ together with $\delta>0$. Then the fundamental solution $\mathcal{E}_{k}(t, s, \xi)$ satisfies the uniform bound

$$
\left\|\mathcal{E}_{k}(t, s, \xi)\right\| \leq C_{k}\left|\Xi_{k}(t, s, \xi)\right|, \quad t \geq s \geq t_{0}, \quad t|\xi|^{2} \leq \delta,
$$

for some constant $C_{k}>0$ depending on $t_{0}, \delta$ and $k$.

Proof. As $\Xi_{k}^{-1}(t, s, \xi)$ is scalar, we conclude that the matrix $\Xi_{k}^{-1}(t, s, \xi) \mathcal{E}_{k}(t, s, \xi)$ satisfies (3.1) with $F_{k}(t, \xi)$ replaced by $F_{k}(t, \xi)-f_{1}^{(k)}(t, \xi) \mathrm{I}$ and, therefore, (3.5) with $\Theta_{k}$ replaced by $\Xi_{k}^{-1} \Theta_{k}$ in all places it occurs. The matrices $\Xi_{k}^{-1} \Theta_{k}$ are uniformly bounded and, hence,

$$
\left\|\mathcal{E}_{k}(t, s, \xi)\right\| \leq\left|\Xi_{k}(t, s, \xi)\right| \exp \left(C^{\prime} \int_{s}^{t}\left\|R_{k+1}(\theta, \xi)\right\| \mathrm{d} \theta\right) .
$$

Furthermore, for $k \geq 2$ the remaining integral is uniformly bounded on this set. 


\section{Lyapunov functionals and parabolic type estimates}

In this section we will partly follow the considerations of Beauchard-Zuazua, [1], and explain how condition (B3) of Section 2 allows to derive parabolic type decay estimates for solutions to the Cauchy problem (1.9). The construction in [1] was inspired by the Lyapunov functionals used by Villani [19].

Lemma 4.1. Assume (B1), (B2), (B3). Then all solutions to (1.9) to Schwartz initial data satisfy the point-wise estimate

$$
\|\widehat{U}(t, \xi)\|^{2} \leq C \mathrm{e}^{-\gamma t[\xi]^{2}}\left\|\widehat{U}_{0}(\xi)\right\|^{2}, \quad[\xi]=|\xi| /\langle\xi\rangle \simeq \min \{|\xi|, 1\}
$$

in Fourier space with constants $C$ and $\gamma$ depending only on the coefficient matrices $A_{k}(t)$ and $B(t)$.

Sketch of proof. The proof follows essentially [1, Sec. 2.2], we will only explain the major steps and necessary modifications to incorporate the time-dependence of matrices. As the problem is $L^{2}$-well-posed and dissipative, it suffices to prove the statement only for $t \geq t_{0}$ with a sufficiently large $t_{0}$.

For a still to be specified selection $\epsilon=\left(\epsilon_{0}, \ldots, \epsilon_{d-1}\right)$ of positive reals, $\epsilon_{j}>0$, we consider the Lyapunov functional

$$
\begin{aligned}
\mathbb{L}_{\epsilon}[\widehat{U} ; t, \xi]=\| \widehat{U}( & t, \xi) \|^{2}+ \\
& +\min \left\{|\xi|,|\xi|^{-1}\right\} \sum_{j=1}^{d-1} \epsilon_{j} \Im\left\langle B(t) A(t, \xi /|\xi|)^{j-1} \widehat{U}(t, \xi), B(t) A(t, \xi /|\xi|)^{j} \widehat{U}(t, \xi)\right\rangle .
\end{aligned}
$$

The uniform Kalman rank condition (B3) implies that for suitable choices of the parameters $\epsilon$ and for $t \geq t_{0}$ the two-sided estimate

$$
\frac{1}{4}\|\widehat{U}(t, \xi)\|^{2} \leq \mathbb{L}_{\epsilon}[\widehat{U} ; t, \xi] \leq 4\|\widehat{U}(t, \xi)\|^{2}
$$

holds true. Therefore, all we have to do is to prove the desired estimate for $\mathbb{L}_{\epsilon}[\widehat{U} ; t, \xi]$, which follows from the differential inequality

$$
\partial_{t} \mathbb{L}_{\epsilon}[\widehat{U} ; t, \xi]+\gamma[\xi]^{2} \mathbb{L}_{\epsilon}[\widehat{U} ; t, \xi] \leq 0
$$

by an application of Gronwall's inequality. Our aim is to find a suitable $\gamma$ and a suitable family $\epsilon$ for this differential inequality to be true.

Formally differentiating $\mathbb{L}_{\epsilon}[\widehat{U} ; t, \xi]$ with respect to $t$ yields the terms considered by [1, Sec. 2.2] together with further terms containing derivatives of the coefficient matrices. The first ones are estimated exactly like in that paper, while the latter ones are bounded by

$$
\mathcal{O}\left(t^{-1}\right) \min \left\{|\xi|,|\xi|^{-1}\right\}\|\widehat{U}(t, \xi)\|^{2}
$$

due to our assumptions. As (4.5) is dominated by $\gamma[\xi]^{2} \mathbb{L}_{\epsilon}[\widehat{U} ; t, \xi]$ whenever $t|\xi| \gtrsim 1$, the desired bound follows on this zone by choosing $\gamma$ small. It remains to consider $t|\xi| \lesssim 1$. Here $A(t, \xi)$ can be treated as small perturbation of $B(t)$ and the diagonalisation scheme and Lemma 3.1 yield already the corresponding bound. 


\section{Estimates for solutions to partially dissipative hyperbolic systems}

$L^{p}-L^{q}$ estimates. First we will conclude parabolic type $L^{p}-L^{q}$ decay estimates. They are a direct consequence of Lemma 4.1 in combination with Hölder's inequality and the boundedness properties of Fourier transform.

Theorem 5.1. Assume (B1), (B2), (B3). Then all solutions to (1.9) satisfy

$$
\|U(t, \cdot)\|_{L^{q}} \leq C(1+t)^{-\frac{n}{2}\left(\frac{1}{p}-\frac{1}{q}\right)}\left\|U_{0}\right\|_{W^{p, r}}
$$

for all $1 \leq p \leq 2 \leq q \leq \infty$ and with $r \geq n(1 / p-1 / q)$.

Proof. For $|\xi| \gtrsim 1$ Lemma 4.1 in combination with Sobolev embedding theorem yields exponential decay under the imposed regularity. Therefore, it is enough to consider bounded $\xi$. But then the estimate follows from Lemma 4.1

$$
\|U(t, \cdot)\|_{L^{q}} \leq\|\widehat{U}(t, \cdot)\|_{L^{q^{\prime}}} \leq\left\|\mathrm{e}^{-\gamma t|\cdot|^{2}}\right\|_{L^{r}}\left\|\widehat{U}_{0}\right\|_{L^{p^{\prime}}} \leq C(1+t)^{-\frac{n}{2}\left(\frac{1}{p}-\frac{1}{q}\right)}\left\|U_{0}\right\|_{L^{p}}
$$

provided $\operatorname{supp} \widehat{U}_{0}(\xi) \subset\{\xi:|\xi| \leq 1\}$ and $\frac{1}{r}=\frac{1}{q^{\prime}}-\frac{1}{p^{\prime}}=\frac{1}{p}-\frac{1}{q}$.

Note, that this a typical parabolic decay estimate. It highlights an underlying diffusive structure. There are related improved decay estimates in this situation, e.g., by assuming moment and decay conditions on the data similar to [4], [5].

Diffusion phenomena. Now we will combine the estimates of the previous section with slightly improved results obtained from the low-frequency diagonalisation. First we construct a parabolic reference problem, whose fundamental solution is given by $\Xi_{2}(t, s, \xi)$ and afterwards we will explain why and in what sense solutions are asymptotically equivalent.

Following Corollary 2.2 it is reasonable to consider the parabolic problem

$$
\partial_{t} w=\nabla \cdot \boldsymbol{\alpha}(t) \nabla w+\boldsymbol{\beta}(t) \cdot \nabla w+\mathrm{i} \gamma(t) w, \quad w\left(t_{0}\right)=w_{0},
$$

for a scalar-valued unknown function $w_{0}$. To relate both problems, we observe that the first row of $\mathcal{E}_{k}(t, s, 0)$ tends to a limit as $t \rightarrow \infty$. This is just a consequence of the integrability of $R_{k+1}(t, 0)$ for $k \geq 2$. We use this to define

$$
W_{k}(s)=\lim _{t \rightarrow \infty} e_{1}^{\top} \mathcal{E}_{k}(t, s, 0) .
$$

It is easy to see that $W_{k}(s)=W_{2}(s)$ for all $k$.

Lemma 5.2. The fundamental solution $\mathcal{E}_{k}(t, s, \xi), k$ sufficiently large, satisfies the estimate

$$
\left\|\mathcal{E}_{k}(t, s, \xi)-\Xi_{k}(t, s, \xi) e_{1} W_{2}(s)\right\| \leq C_{k}(1+t)^{-\frac{1}{2}}, \quad t \geq s \geq t_{0},
$$

uniformly on $|\xi| \leq 1$.

Proof. We make use of a constant $\delta>0$, to be fixed later on, to decompose the extended phase space into several zones. 
Part 1. If $t|\xi|^{2} \geq \delta \log t$ with $\delta$ chosen large enough, both terms can be estimated separately by $\exp \left(-\tilde{\gamma} t|\xi|^{2}\right)$ for some constant $\gamma$. This follows for the first one by Lemma 4.1 and for the second by the parabolicity of (5.2) in consequence of Corollary 2.2, But then

$$
\mathrm{e}^{-\tilde{\gamma} t|\xi|^{2}} \leq \mathrm{e}^{-\tilde{\gamma} \delta \log t}=t^{-\tilde{\gamma} \delta} \lesssim t^{-\frac{1}{2}}, \quad \tilde{\gamma} \delta \geq \frac{1}{2} .
$$

Part 2. If $t|\xi|^{2} \leq \delta$ for some $\delta$, we use the results from the asymptotic integration of the diagonalised system. First, we claim that $\Xi_{k}^{-1}\left(t, t_{0}, \xi\right) e_{1}^{\top} \mathcal{E}_{k}\left(t, t_{0}, \xi\right)$ converges locally uniform in $\xi$ as $t \rightarrow t_{\xi}$ for $t_{\xi}|\xi|^{2}=\delta$. To see this, we use the Neumann series representation of $\Xi_{k}^{-1}\left(t, t_{0}, \xi\right) \mathcal{E}_{k}\left(t, t_{0}, \xi\right)$ (i.e., (3.5) with $\Theta_{k}$ replaced by $\Xi_{k}^{-1} \Theta_{k}$ ) multiplied by $e_{1}^{\top}$

$$
\begin{aligned}
\Xi_{k}^{-1}\left(t, t_{0}, \xi\right) e_{1}^{\top} & \mathcal{E}_{k}\left(t, t_{0}, \xi\right)=e_{1}^{\top}+\sum_{\ell=1}^{\infty} \mathrm{i}^{\ell} \int_{t_{0}}^{t} e_{1}^{\top} R_{k+1}\left(t_{1}, \xi\right) \int_{t_{0}}^{t_{1}} \Xi_{k}^{-1}\left(t_{1}, t_{2}, \xi\right) \Theta_{k}\left(t_{1}, t_{2}, \xi\right) R_{k+1}\left(t_{2}, \xi\right) \\
& \times \int_{t_{0}}^{t_{2}} \cdots \int_{t_{0}}^{t_{\ell-1}} \Xi_{k}^{-1}\left(t_{\ell-1}, t_{\ell}, \xi\right) \Theta_{k}\left(t_{\ell-1}, t_{\ell}, \xi\right) R_{k+1}\left(t_{\ell}, \xi\right) \mathrm{d} t_{\ell} \cdots \mathrm{d} t_{2} \mathrm{~d} t_{1}
\end{aligned}
$$

and use the Cauchy criterion. We denote the resulting limit as $W_{k}(s, \xi)$ and observe that it coincides with $W_{k}(s)$ for $\xi=0$. Next, we show the estimates

$$
\left\|W_{k}(s, \xi)-W_{k}(s)\right\| \lesssim|\xi|
$$

and

$$
\left\|e_{1}^{\top} \mathcal{E}_{k}(t, s, \xi)-\Xi_{k}(t, s, \xi) W_{k}(s, \xi)\right\| \lesssim t^{-\frac{1}{2}}
$$

The first of these estimates follows as uniform limit for estimates of the difference

$$
\Xi_{k}^{-1}(t, s, 0) e_{1}^{\top} \mathcal{E}_{k}(t, s, 0)-\Xi_{k}^{-1}(t, s, \xi) e_{1}^{\top} \mathcal{E}_{k}(t, s, \xi) .
$$

Indeed, using again the Neumann series we see that the first terms are equal. The second terms are reduced to the estimate

$$
\left\|R_{k+1}\left(t_{1}, \xi\right)-R_{k+1}\left(t_{1}, 0\right)\right\| \lesssim|\xi|\left(t_{1}^{-k}+|\xi|^{k}\right), \quad t_{1} \geq t_{0}
$$

following directly from the definition of the $\mathcal{P}\{k+1\}$-classes. Therefore,

$$
\left\|\Xi_{k}^{-1}(t, s, 0) e_{1}^{\top} \mathcal{E}_{k}(t, s, 0)-\Xi_{k}^{-1}(t, s, \xi) e_{1}^{\top} \mathcal{E}_{k}(t, s, \xi)\right\| \lesssim|\xi| \int_{s}^{t}\left(\theta^{-k}+|\xi|^{k}\right) \mathrm{d} \theta+\text { l.o.t. }
$$

and the right-hand side is uniformly bounded by $|\xi|$. Taking limits proves the estimate. The second estimate is similar. Again using the Neumann series we see that this difference can be estimated by

$$
\begin{aligned}
\left\|\Xi_{k}^{-1}(t, s, \xi) e_{1}^{\top} \mathcal{E}_{k}(t, s, \xi)-W_{k}(s, \xi)\right\| & \\
& \lesssim \int_{t}^{t_{\xi}}\left\|R_{k+1}(\tau, \xi)\right\| \exp \left(\int_{t_{0}}^{t_{\xi}}\left\|R_{k+1}(\theta, \xi)\right\| \mathrm{d} \theta\right) \mathrm{d} \tau \lesssim t^{-\frac{1}{2}}
\end{aligned}
$$

due to $\left\|R_{k+1}(t, \xi)\right\|=\mathcal{O}\left(t^{-\frac{3}{2}}\right)$ for $k \geq 2$ and $t|\xi|^{2} \leq \delta$.

Combining both of the above estimates and using that the other rows in $\mathcal{E}_{k}$ are exponentially decaying we get

$$
\begin{aligned}
\left\|\mathcal{E}_{k}(t, s, \xi)-\Xi_{k}(t, s, \xi) e_{1} W_{k}(s)\right\| \leq & \left\|\mathcal{E}_{k}(t, s, \xi)-\Xi_{k}(t, s, \xi) e_{1} W_{k}(s, \xi)\right\| \\
& +\left\|\Xi_{k}(t, s, \xi) e_{1} W_{k}(s, \xi)-\Xi_{k}(t, s, \xi) e_{1} W_{k}(s)\right\| \\
& \lesssim t^{-\frac{1}{2}}+\mathrm{e}^{-\tilde{\gamma} t|\xi|^{2}}|\xi| \lesssim t^{-\frac{1}{2}} .
\end{aligned}
$$


Part 3. It remains to consider the logarithmic gap between both parts, i.e., $\delta \leq t|\xi|^{2} \leq \delta \log t$. Here we use that for $k$ sufficiently large the remainder term $R_{k+1}(t, \xi)$ decays as $t^{-k-1+\epsilon}$, while the polynomial growth rate of $\Xi_{k}^{-1}(t, \xi)$ is independent of $k$ for large $k$. Choosing $k$ large enough, the Neumann series argument gives

$$
\left\|\Xi_{k}^{-1}(t, s, \xi) e_{1}^{\top} \mathcal{E}_{k}(t, s, \xi)-\tilde{W}_{k}(s, \xi)\right\| \lesssim \int_{t}^{\tilde{t}_{\xi}}\left\|\Xi_{k}^{-1}(\theta, s, \xi) R_{k+1}(\theta, \xi)\right\| \mathrm{d} \theta \lesssim t^{-\frac{1}{2}}
$$

with $\tilde{t}_{\xi}$ defined by $\tilde{t}_{\xi}|\xi|^{2}=\delta \log \tilde{t}_{\xi}$ and

$$
\tilde{W}_{k}(s, \xi)=\lim _{t \rightarrow \tilde{t}_{\xi}} \Xi_{k}^{-1}(t, s, \xi) e_{1}^{\top} \mathcal{E}_{k}(t, s, \xi) .
$$

The existence of the latter limit follows for large $k$ and again $\tilde{W}_{k}(s, \xi)-W_{k}(s)$ coincides up to order $\mathcal{O}(|\xi|)$.

To obtain a statement in terms of the original equation, we introduce $K(t, \xi)=M(t) N_{2}(t, \xi) e_{1}$. By definition we have $K(t, \xi) \in(\mathcal{P}\{0\}+\mathcal{P}\{1\}+\mathcal{P}\{2\}) \otimes \mathbb{C}^{d}$. We define further $w_{0}=W U_{0}$ in such a way that we cancel the main term of the solution within $\mathcal{Z}_{\text {ell }}\left(c_{k}\right) \cap\{t|\xi| \leq \delta\}$, i.e., we define

$$
\widehat{w}_{0}=W_{2}\left(t_{0}\right) N_{2}^{-1}\left(t_{0}, \xi\right) M^{-1}\left(t_{0}\right) \mathcal{E}\left(t_{0}, 0, \xi\right) \chi(\xi) \widehat{U}_{0}
$$

with $\chi(\xi) \in C_{0}^{\infty}\left(\mathbb{R}^{n}\right), \chi(\xi)=1$ near $\xi=0$ and supp $\chi \subset B_{c_{2}}(0)$. Then the estimate of Lemma 5.2 implies the following statement. The logarithmic term is caused by comparing $\Xi_{k}(t, s, \xi)$ with $\Xi_{2}(t, s, \xi)$.

Theorem 5.3. Assume (B1), (B2), (B3) and let $U(t, x)$ be solution to (1.9). The the solution $w(t, x)$ to (5.2) with data given by (5.16) satisfies

$$
\|U(t, \cdot)-K(t, \mathrm{D}) w(t, \cdot)\|_{L^{2}} \leq C^{\prime}(1+t)^{-\frac{1}{2}} \log (\mathrm{e}+t)\left\|U_{0}\right\|_{L^{2}}
$$

\section{Concluding remarks}

(1). The logarithm in (5.17) is most likely not sharp, while we conjecture that the rate $(1+t)^{-1 / 2}$ is sharp as long as no further symmetry conditions are satisfied.

(2). Assumption (B1) may be strengthend by assuming that $B(t) \geq 0$. Under this assumption the problem has an additional symmetry in Fourier space,

$$
(A(t, \xi)+\mathrm{i} B(t))^{*}=A(t,-\xi)-\mathrm{i} B(t)=-(A(t, \xi)+\mathrm{i} B(t))
$$

and it follows that the fundamental solution satisfies $\mathcal{E}(t, s,-\xi)=\mathcal{E}(t, s, \xi)^{*}$. In consequence, the coefficient $\boldsymbol{\beta}(t)$ of the associated parabolic problem is purely imaginary, the estimate in (5.7) is of order $|\xi|^{2}$ and similar for the following estimates. Finally, the estimate (5.17) improves by half an order. 


\section{References}

[1] K. Beauchard and E. Zuazua. Large time asymptotics for partially dissipative hyperbolic systems. Arch. Ration. Mech. Anal., 199 (2011) no. 1, 177-227.

[2] R. Chill and A. Haraux. An optimal estimate for the difference of solutions of two abstract evolution equations. J. Differential Equations, 193 (2003), no. 2, 385-395.

[3] Ling Hsiao and Tai-Ping Liu. Convergence to nonlinear diffusion waves for solutions to a system of hyperbolic conservation laws with damping. Commun. Math. Phys., 143 (1992) 599-605.

[4] R. Ikehata. Decay estimates by moments and masses of initial data for linear damped wave equations. Int. J. Pure Appl. Math. 5 (2003) 77-94.

[5] R. Ikehata. Improved decay rates for solutions to one-dimensional linear and semilinear dissipative wave equations in all space. J. Math. Anal. Appl. 277 (2003) 555-570.

[6] R. Ikehata and K. Nishihara. Diffusion phenomenon for second order linear evolution equations. Stud. Math., 158 (2003) 153-161.

[7] K. Jachmann and J. Wirth. Diagonalisation schemes and applications. Ann. Mat. Pura Appl., 189 (2010) 571-590.

[8] S. Kawashima and Y. Shizuta. Systems of equations of hyperbolic-parabolic type with application to the discrete Boltzmann equation. Hokkaido Math. J., 14 (1985) 249-275.

[9] A. Milani and Han Yang. $L^{1}$ decay estimates for dissipative wave equations. Math. Methods Appl. Sci., 24 (2001) 319-338.

[10] T. Narazaki. $L^{p}-L^{q}$ estimates for damped wave equations and their applications to semi-linear problem. J. Math. Soc. Japan, 56 (2004) 585-626.

[11] K. Nishihara. Asymptotic behaviour of solutions of quasilinear hyperbolic equations with linear damping. J. Differential Equations, 137 (1997) 384-395.

[12] K. Nishihara. $L^{p}-L^{q}$ estimates of solutions to the damped wave equation in 3-dimensional space and their application. Math. Z., 244 (2003) 631-649.

[13] P. Radu, G. Todorova and B. Yordanov. Diffusion phenomenon in Hilbert spaces and applications. J. Differential Equations, 250 (2011) 4200-4218.

[14] M. Ruzhansky and J. Wirth. Dispersive estimates for T-dependent hyperbolic systems. Rend. Sem. Mat. Univ. Pol. Torino, 66 (2008) 339-349. Corrigendum: 68 (2010), 93.

[15] M. Ruzhansky and J. Wirth. Dispersive estimates for $t$-dependent hyperbolic systems. $J$. Differential Equations 251 (2011) 941-969.

[16] J. Wirth. Wave equations with time-dependent dissipation. II. J. Differential Equations, 232 (2007) 74-103. 
[17] J. Wirth. Block-diagonalisation of matrices and operators. Lin. Alg. Appl., 431 (2009) 895902.

[18] J. Wirth. Energy inequalities and dispersive estimates for wave equations with time-dependent coefficients. Rend. Mat. Univ. Trieste, 42 Suppl. (2010) 205-219.

[19] C. Villani. Hypocoercivity. Mem. Amer. Math. Soc., 202 (2009) no. 950. 